\title{
Pareto Set Computation in Convex Multi-objective Design using Adaptive Response Surface Method (ARSM)
}

\author{
Miguel A. Panesso ${ }^{1}$, Camilo J. Cruz', Juan C. Bohorquez², Luis E. Muñóz ${ }^{1}$, Néstor M. Peña ${ }^{2}$, Fredy E. Segura ${ }^{2}$ \\ ${ }^{1}$ Universidad de los Andes, Mechanical Engineering Department, Cra $1^{\text {a }}$ No $18^{a}-12$ Bogotá, Colombia \\ ${ }^{2}$ Universidad de los Andes, Electrical and Electronic Engineering Department, Cra $1^{a}$ No $18^{a}-12$ Bogotá, Colombia
}

\begin{abstract}
Optimal design problems normally involve high dimensional design spaces and multiple objective functions. Depending on the complexity of the model, the time required to explore the design space could become excessive. This paper describes the calculation of the Pareto-optimal set based on adaptive surface methodology (ARSM) in order to reduce simulation times given a finite element analysis (FEA) simulation model. The Paretooptimal strategy consists in the solution of a set of different single-objective problems. Each of this points is found via ARSM. The implementation of ARSM aims to use a few initial simulation points to approximate accurately the set of single-objective functions required. The methodology reduces significantly the number of points required to compute the efficient set compared to other strategies (e.g the exhaustive method), proving to reduce the simulation time of a computationally intensive model.
\end{abstract}

\section{Introduction}

Computational numeric simulations of complex geometric models have become essential in the mechanical design process of the last decades. The implementation of such methods make it possible to solve problems in which the solution by an analytical formulation is not possible.

The field of optimal design has been greatly benefited by its implementation given the relation between model complexity and time processing improvement. In a typical design optimization where a selected design space is constrained by design restrictions, it's desired to find the best solution according to design requirements. However, even with the implementation of computational aided calculations, an exhaustive exploration of the solution space leads to extended simulation times that are not practical, particularly for large computational intensive models.

One of the proposed tools to solve this kind of problems is the response surface method (RSM) [1]. It consists of the combination of several techniques including mainly a design of experiments (DOE) and model fitting. The main purpose of RSM is to find an approximate form of the objective function, which allows the use of few sample points instead of making an exhaustive exploration.

\section{Response Surface Method Review}

According to Gosavi [2], RSM begins by choosing the points to be calculated from the design space. The selection of these points should be performed in order to minimize the number of simulations with the best possible quality of the fitting model. Several classic DOE techniques have been widely developed to achieve this goal, including factorial designs, central composite designs, Lat in hypercube designs and low discrepancy sequences, just to name a few.

After the selection of the design variable space, an expected behaviour of the response represented by a meta-model is selected. In the most classic approach [1], this behaviour is assumed for simplicity of second order or even higher order degree polynomial, depending on the model's complexity. An appropriate fitting method for that meta-model is used to generate the response surface. Techniques such as linear regression, splines regression and neural networks are commonly used as fitting methods. Approximation quality can be proved with a statistical test. For least squares regression method, the parameter $\mathrm{r}^{2}$ is normally used. Nevertheless, $\mathrm{r}^{2}$ misleads to good results when the assumed polynomial is high order or there are several design para meters. Further validation tests can be found in [3], [4] and [5].

In synthesis, the classical RSM method involves DOE, meta-model selection, fitting techniques and validation trough statistical methods.

Avoiding to restrict the response to a predefined set of points in the design space, new approaches have been developed in the last years that iteratively update the design space with the goal of achieving an optimum. The update consists in finding the minimum of the first response surface obtained by the typical techniques described before with an optimization algorithm, adding 
the design variables of the minimum point to the initial design space and removing the point (or points, given a threshold) with the highest objective function value. The methodology was first proposed by Wang ([6], [7]) with the name of Adaptive Response Surface Methodology (ARSM).

The method proposed by Wang involves the domain reduction of each design variable, which its time consuming given the minimization process for both upper and lower limit. Steenackers [8] proposed a revised method that does not involve the reduction of the design space, but rather a strategy called "pan and zoom" (for more details refer to [8]). Compared to Wang's method, the pan and zoom method showed a reduced time per iteration as well as the total number of iterations required to converge to the minimum. For further examples using adaptive method, refer to [9] - [14].

The present study is based on the ASRM methods proposed by both Wang and Steenackers to explore the solution space of a finite element analysis (FEA) 2D simu lation. In addition to previous works, this paper aims to apply the method to a multivariable design space and extend it to a multiple objective optimization functions.

Particularly, the study proposes the implementation of the ARSM to a single objective function that contains the information of a multiple objective problem using weights. Given a defined number of weight co mbinations to be determined, each set of them requires an implementation of the algorithm. The final result is a Pareto-set plot efficiently obtained by the use of the ARSM, avoiding an exhaustive method which involves the simulation of the complete design space. The consequence of this is a significant reduction in simulation times (without even taking into account the fact that for several design variables and objectives, the enumeration method fails to produce a result in reas onable time).

\section{Methodology - ARSM}

This paper implements an ARSM method jointly with the Pareto set computation of a convex multi-objective design problem. The proposed approach is based on the formulation and solution of a set of single-objective problems, where the single objective function is obtained as weighted sum of the objective functions of the orig inal problem ([18], [19]). Weights are assigned to each single function as follows:

$$
F=w_{1} f_{1}+w_{2} f_{2}
$$

Where $\mathrm{F}$ is a linear combination of each objective function $\mathrm{f}_{\mathrm{i}}$; and $\mathrm{w}_{\mathrm{i}}$ is the given weight of each function, being the sum of weights equal to 1 . The minimum of this function is calculated using ARSM for each pair of weights. The aim is to obtain an approximation of the Pareto set using considerably less points than in the case of simple numeration of an exhaustive explored design space.

Particularly, the ARSM method is based on the "Pan and Zoom" method [8]. It consists in the following steps:
1- The method begins by the selection of points from the design space. For this study, a Sobol Low discrepancy sequence ([15], [16]) was used to explore uniformly the space. The Sobol set was configured to also include the boundary of the design space.

2- The selected points are simulated to obtain the real value of the objective function.

3- The obtained objective function values are fitted with a second order degree polynomial.

4- The response surface is minimized using a local optimization algorithm. In this case, an algorithm from a commercial software was used (i.e. Matlab), which corresponds to Nelder-Mead method [17].

5- The calculated minimum is added to the initial set of points which represents a promising search direction from the real objective function minimum. Simultaneously, the point with the highest objective function value is erased. This step reduces the design space which increase the quality of the response surface in the minimal region.

6- With a user defined convergence criteria, the objective function value obtained is compared to the response surface value. If the difference achieves the criteria, the minimum of the surface is taken as the real objective function. In the opposite case, the method returns to step 2 and repeat the process until the criteria is met.

The aforementioned methodology is repeated for each pair of weights used to construct an approximation of the Pareto set.

An exhaustive exploration was performed to determine the real Pareto set. The set was adjusted to a bi-exponential regression. The same fit was applied to the approximated Pareto set found by ARSM.

To compare the accuracy of the approximation to the complete design space Pareto front, the determination coefficient $\mathrm{R}^{2}$ [4] was used:

$$
\begin{gathered}
S S_{R E S}=\sum\left(y_{i}-f_{i}\right)^{2} \\
S S_{T O T}=\sum\left(y_{i}-y_{\text {mean }}\right)^{2} \\
R^{2}=1-S S_{R E S} / S S_{T O T}
\end{gathered}
$$

Where $y_{i}$ is the $i$-th value of the Pareto front approximation using ARSM, $\mathrm{y}_{\text {mean }}$ is the mean value from the Pareto front approximation using ARSM, and $\mathrm{f}_{\mathrm{i}}$ is the i-th value from the complete design space Pareto

\section{Case of Study}

\subsection{Problem formulation}

In order to prove the scope of the implementation, a 2D cantilever beam was simulated including an elastomeric interface embedded on a polymeric matrix instead of a common fixed support. The simulated geometry is shown on Figure 1. For the sake of simp licity, all materials were considered within their linear elastic range. Two design 
parameters where considered for each simulation, where P1 is the initial thickness of the elastomeric interface and $\mathrm{P} 2$ is the gap between the polymeric matrix and the beam. A plane stress formulation was implemented.

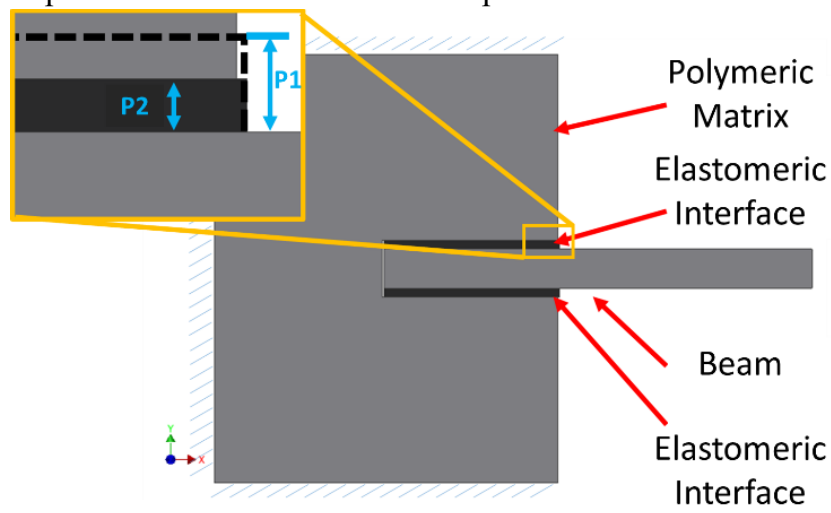

Figure 1. Simulated geometry

Figure 2 presents the load condition of the simulation. Given the nature of the case, a $10 \mathrm{~N}$ load was applied at the beam's tip. The supports were fixed in the upper, lower and rear sides of the polymeric matrix. Finally, the elastomeric interface pre-stress generated by the variation of P1 and P2 was represented as a distributed load on the contact zone between the elastomeric interfaces and the upper/lower beam sides.

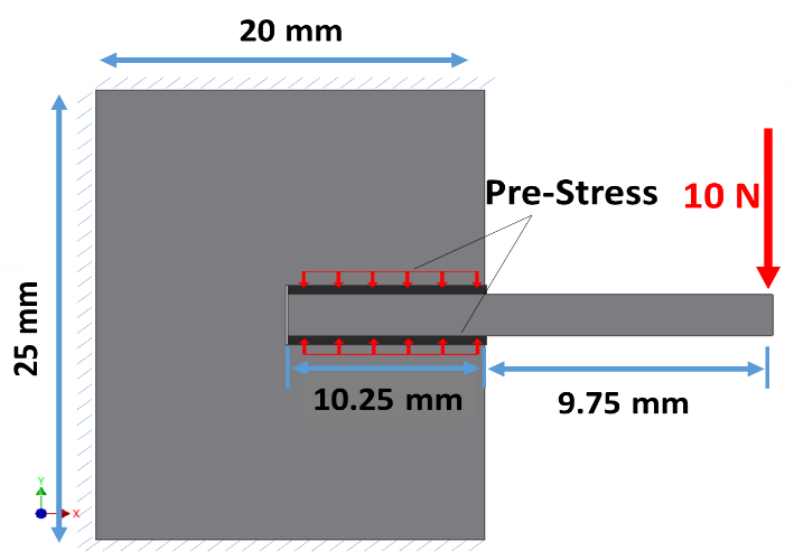

Figure 2. Dimensions, loads and supports

The model was conceived to simplify the geo metry of a measurement instrument support. The optimal design problem consists in minimizing both natural frequency of the system and deflection at the beam's tip. The parameters [P1, P2] are related to the elastomeric interface pre-stress. Therefore, both objective functions will be dependent from the distributed load generated by the variation of $\mathrm{P} 1$ and $\mathrm{P} 2$. Formally,

Subject to:

$$
\min d(P 1, P 2) \quad \min f(P 1, P 2)
$$

$$
\begin{gathered}
510 \mu m<P 1<600 \mu m \\
500 \mu m<P 2<590 \mu m \\
P 1 / P 2>1
\end{gathered}
$$

The deflection will be reduced with a greater distributed load, given that the elastomeric interfaces are able to support a greater reacting momentum generated by the load force at the beam's tip. In the other hand, a greater distributed load will increase the system's natural frequency due to an increment in the stiffness.

\subsection{Simulation conditions}

From the FEA point of view, the mesh used for the polymeric matrix and the elastomeric interface was parameterized as function of both variables [P1, P2] to keep the element size according to the change of geometry. This relationship ratio between element size and parameter size was determined after a convergence study. An 8 node high order quadratic element was used in order to accurately capture high deformation behavior. Table 1 shows the summarized information for each simulation component.

Table 1. FE Simulation characteristics

\begin{tabular}{|c|c|c|c|c|}
\hline Component & $\begin{array}{c}\text { Young } \\
\text { Modulus } \\
{[\text { GPa] }}\end{array}$ & $\begin{array}{c}\text { Density } \\
{[\mathrm{kg} / \mathrm{m} 3]}\end{array}$ & $\begin{array}{c}\text { Element } \\
\text { Size [m] }\end{array}$ & $\begin{array}{c}\text { Element } \\
\text { Type }\end{array}$ \\
\cline { 1 - 4 } $\begin{array}{c}\text { Polymeric } \\
\text { Matrix }\end{array}$ & 2.7 & 1200 & $6.40 \mathrm{E}-04$ & \multirow{2}{*}{ PLANE } \\
\cline { 1 - 4 } Beam & 57 & 2500 & $1.47 \mathrm{E}-04$ & \\
\cline { 1 - 4 } $\begin{array}{c}\text { Elastomeric } \\
\text { Interface }\end{array}$ & 0.025 & 1000 & $1.47 \mathrm{E}-04$ & \\
\hline
\end{tabular}

In terms of contacts, all the contacts between the polymeric matrix and the elastomeric interfaces were considered as bonded contacts. This resembles an adhesive union between the elements. All the contacts between the beam and the elastomeric interfaces were simulated as an infinite friction coefficient union. Given that the load is applied in the vertical direction (and the fact that the problem is defined with a plane stress formulation), the behavior of this contact allows to accurately study the deflection at the beam's tip, and first resonant frequency. The vibration mode corresponding to the first resonant frequency is shown in Figure 3.

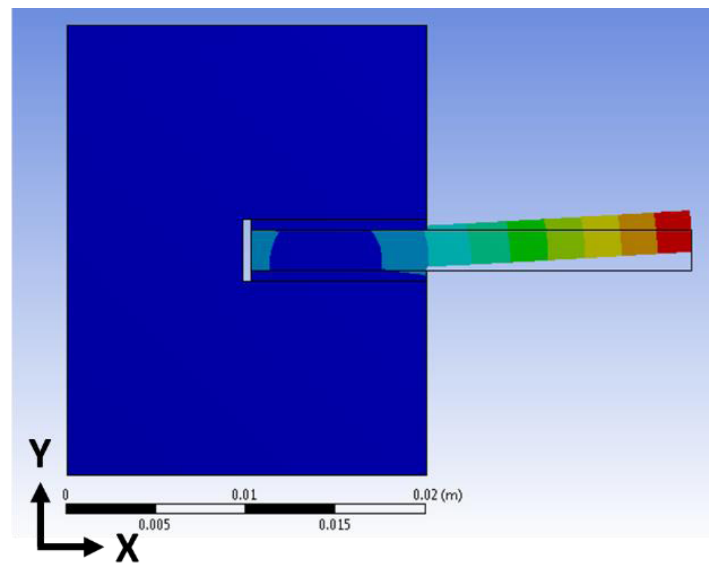

Figure 3. First resonance frequency

\subsection{Simulation range}


As explained in the section 3.2, the contacts between the elastomeric interfaces and the surface were modeled as an infinite friction coefficient union. Therefore the parameters [P1, P2] were constrained to be P1 > P2 in order to simulate only compression conditions for the elastomeric interfaces.

In addition to the compression, the ranges for [P1, P2] were assumed for a maximal $20 \%$ deformation of the elastomeric interface. In this way, the maximal distributed load applied by the pre-stress will be $615 \mathrm{~N}$. Table 2 shows the limits defined for each variable

Table 2. Variable simulation range

\begin{tabular}{|c|c|c|c|}
\hline Limit & P1 [mm] & P2 [mm] & Pre-stress Load $[\mathbf{N}]$ \\
\hline Min & 0.51 & 0.5 & 10 \\
\hline Max & 0.6 & 0.59 & 615.2 \\
\hline
\end{tabular}

\section{Results}

Figure 4 shows an example of a normalized surface corresponding to the single-objective problem for a given pair of weights as a result of the second order regression.

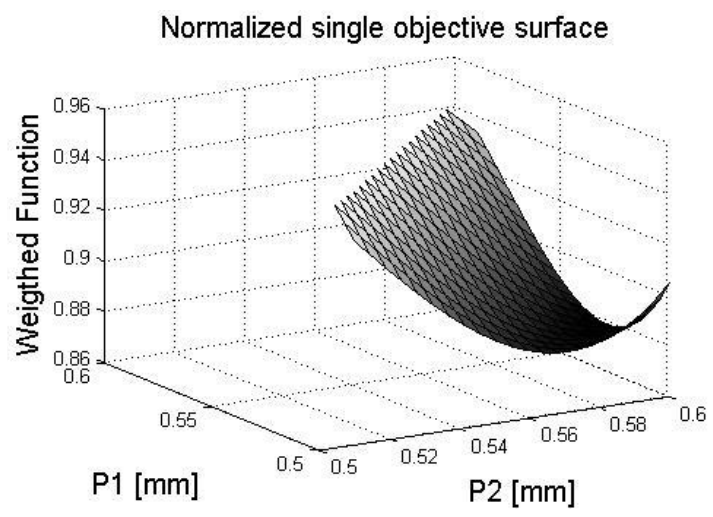

Figure 4. Normalized single objective surface

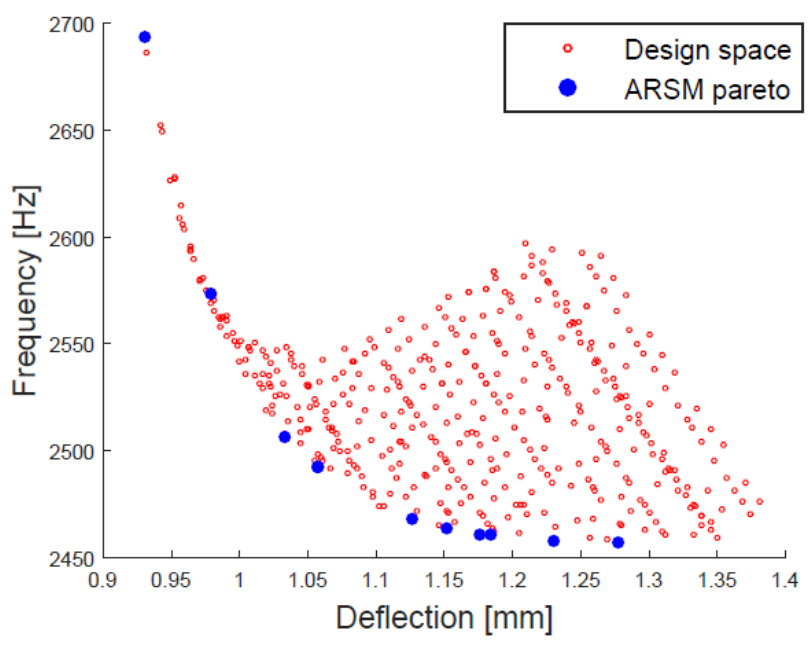

Figure 5 Design space calculated by exhaustive method (dots) and ARSM Optimal Pareto-set (stars)
Figure 5 shows the multi-objective plot between deflection and frecuency considering the points of the completely simulated design space and those obtained by the ARSM for 10 pair of weights. A total of 418 points where used in the first case, and it took 55 evaluations of the simulation to compute the pareto set in the second case.

Figure 6 shows the comparis son between the fitting of a full explored pareto front (solid line), and the ARSM approximations obtained with different in itial simulation points (n). As expected, a higher number of initial points will produce a betterquality Pareto front approximation.

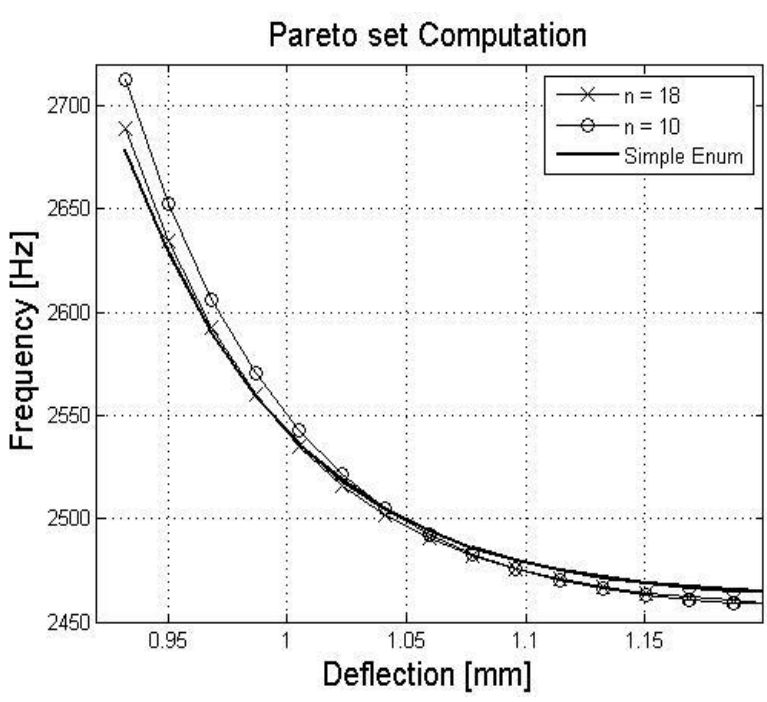

Figure 6. Pareto front comparison between ARSM approximation and exhaustive method.

This observation is confirmed numerically by calculating $\mathrm{R}^{2}$ determination coefficient named in section 2. Table 3 resumes the simulations results for the three cases (exhaustive method, 10 in itial points and 18 initial points). There exist a tradeoff between the quality of the approximation and the initial number of points. In any case, both ARSM approximations required only a fraction of the computational effort used to compute the Pareto set.

Table 3. Simulations results

\begin{tabular}{|c|c|c|c|}
\hline \multicolumn{4}{|c|}{ EXHAUSTIVE METHOD } \\
\hline \multicolumn{4}{|c|}{418} \\
\hline \multicolumn{4}{|c|}{ ARSM METHODOLOGY } \\
\hline $\begin{array}{c}\text { Number of } \\
\text { initial points in } \\
\text { ARSM }\end{array}$ & $\begin{array}{c}\text { ARSM } \\
\text { Iterations }\end{array}$ & $\begin{array}{c}\text { Total } \\
\text { simulated } \\
\text { points }\end{array}$ & $\begin{array}{c}\mathbf{R}^{2} \\
\text { determination } \\
\text { coefficient }\end{array}$ \\
\hline 10 & 32 & 42 & 0.9756 \\
\hline 18 & 37 & 55 & 0.9939 \\
\hline
\end{tabular}

\section{Conclusions}

The methodology shows a significant improvement in computation times compared to the simulation of the complete design space followed by the exhaustive 
method of simple enumeration, thus saving time in a highly computational intensive model.

In this case, the computation of the Pareto-optimal set for a given set of weights represented only $13 \%$ of the total simulation time requiered for the simulation of the complete design space: 14 hours for exahustive method, 1 hour 49 minutes for 18 initial ARSM points and 1 hour 24 minutes for 10 in itial ARSM points; with a marg inal compromise in accuracy. In the 18 points case, the determination coefficient was 0.9939 with respect to the exhaustive Pareto-set, while in the 10 points case, it was 0.9756 .

\section{Acknowledgements}

We acknowledge B. Moreno, F. De Milleri and H. Rojas for their assistance, technical discussion and analys is in this work. This project was funded by COLCIENCIAS, CODENSA S.A E.S.P and Universidad de los Andes according to the RC. No. 0582-2013 agreement.

\section{References}

1. R. H. Myers, D. C. Montgomery and C. M. Anderson-Cook, Response Surface Methodology: Process and Product Optimization using Designed Experiments, John Wiley \& Sons., 2009

2. Gosavi, "Parametric Optimization: Response Surfaces And Neural Networks," in SimulationBased Optimization: Parametric Optimization Techniques and Reinforcement Learning, Rolla, Mis souri, USA, Springer, 2015, pp. 37-46.

3. A. Cameron, A. G. Windmeijer, H. Gramajo, D. Cane and C. Khosla, "An R-squared measure of goodness of fit for some common nonlinear regression models," Journal of Econometrics, vol. 77, no. 2, pp. 329-342, 1997.

4. J. P. Kleijnen and R. G. Sargent, "A methodology for fitting and validating metamodels in Simulation," European Journal of Operation Research, pp. 14-29, 2000.

5. S. Weisberg, Applied Linear Regression, Wiley, 2005.

6. G. G. Wang, Z. Dong and P. Aitchison, "Adaptative Response Surface Method - A Global Optimization Scheme for Approximation-based Design Problems," Journal of Engineering Optimization, pp. 707-734, 2001.

7. G. G. Wang, «Adaptive response surface method using inherited Latin hypercube design points,» ASME Journal of Mechanical Design, vol. 1, $\mathrm{n}^{\circ}$ 125, pp. 210-220, 2003.
8. G. Steenackers, F. Presezniak and P. Guillaume, "Development of an adaptive response surface method for optimization of computation-intensive models," Computers \& Industrial Engineering, Elsevier, pp. 847-855, 2009.

9. L. Xiaojia y N. Fangfei, «New response surface model and its applications in aerodynamic optimization of axial compressor blade profile,» Front. Energy Power Eng. China, vol. 2, n 4, pp. 541-549, 2008.

10. J. Lee, S. Shin y S. Kim, «An Optimal Design of Marine Systems based on Neuro-Response Surface Method,» de 10th International Conference on Natural Computation, Xiamen, 2015.

11. N. Roussouly, F. Petitjean y M. Salaun, «A new adaptive response surface method for reliability analysis,» Probabilistic Engineering Mechanics, vol. 1, no 32, pp. 103-115, 2013.

12. S. Chakraborty y S. Arunabh, «Adaptive response surface based efficient Finite Element Model Updating,» Finite Elements in Analysis and Design, vol. 1, no 80, pp. 33-40, 2014.

13. X. S. Nguyen, A. Sellier, F. Duprat y G. Pons, "Adaptive response surface method based on a double weighted regression technique,» Probabilistic Engineering Mechanics, vol. 1, $\mathrm{n}^{\mathrm{o}}$ 24, pp. 135-143, 2009.

14. W. Y. L. G. Hu y Z. Hua, «Optimization of sheet metal forming processes by adaptive response surface based on intelligent sampling method,» journal of materials processing technology, vol. $1, \mathrm{n}^{\circ}$ 197, pp. 77-88, 2008.

15. T. Santner, B. Williams and N. W., The Design and Analysis of Computer Experiments, Springer, 2003.

16. P. Bratley and B. L. Fox, "Algorithm 659 Implementing Sobol's Quasirandom Sequence Generator," ACM Transactions on Mathematical Software, vol. 14, no. 1, pp. 88-100, 1988.

17. J. C. Lagarias, J. A. Reeds, M. H. Wright and P. E. Wright, "Convergence Properties of the NelderMead Simplex Method in Low Dimensions," SIAM Journal of Optimization, vol. 9, no. 1, pp. 112-147, 1998.

18. G. Mastinu, M. Gobbi and C. Miano, Optimal Design of Complex Mechanical Systems with Applications to Vehicle Engineering, Springer, 2006.

19. P. Y. Papalambros and D. J. Wilde, Principles of Optimal Design: Modeling and Computation, Cambridge University Press, 2000. 